\title{
Real-time Air-Sense Module for Monitoring Enclosed Livestock Housing
}

\author{
Manal Beshay ${ }^{1}$, Lihua Zhang ${ }^{1}$, Marvin Lav', Robert Christenson ${ }^{2}$ \\ 1 Intelligent Optical Systems, Inc., 2520 W. 237th Street, Torrance, California, 90505, USA \\ ${ }^{2}$ CTB, Inc. (Chore-Tronics), 611 N. Higbee Street, Milford, Indiana, 46542, USA \\ mbeshay@intopsys.com
}

\begin{abstract}
:
Animal barns need ventilation systems that effectively measure temperature, humidity, ammonia, and other gases. Reliable sensors that accurately measure these parameters will contribute to a healthy environment for the animals and the workers, while not wasting energy and money on over-ventilation. Current systems are either state-of-the-art instruments for field use that are precise and accurate but limited by their cost, size, and demands on power and labor; or inexpensive test kits and point sensors that perform single measurements with acceptable accuracy and sensitivity, but are unsuited to livestock operations. In addition to the costs of both over- and under-ventilation-overuse of energy, and adverse effects on animal health and productivity, respectively - there are important effects on worker health. This extends to residential areas near livestock facilities, which can be exposed to gas levels that cause not only discomfort but also long- and short-term health damage, including eye, nose, and throat irritation, headaches, dizziness, and fatigue. Long-term effects, usually the result of extended, cumulative exposure, can include respiratory disease, heart disease, and cancer, and can be severely debilitating or even fatal. Building on the successful results of our prior development, we have continued advancing the Air-Sense modules by: (1) finalizing signal processing to model the sensor hysteresis resulting from long-term aging in livestock environments, including exposure to volatile organic compounds (VOCs) as potential interferents; (2) designing the communication software to meet the target signal output for a ventilation system interface; and (3) integrating multiplexed temperature and humidity measurement into the unit output.
\end{abstract}

Key words: sensor, optical, cost-effective, gas phase, ventilation

\section{Background}

There is a serious need for monitoring the air in enclosed livestock housing. While measurement technologies such as chemiluminescence, open path Fourier transform infrared (OP-FTIR), and UV differential optical spectroscopy are robust and accurate, their cost and need for routine maintenance exclude them from the budgetconstrained livestock barn market. Mid-range priced technologies such as electrochemical handheld sensors are not suitable for livestock environments because their reactive surfaces are poisoned by ambient VOCs [1]. Colorimetric pull tubes are frequently used to measure ammonia levels with satisfactory accuracy and cost, but cannot deliver the real-time coverage and ventilation rate synchronization needed for effective barn monitoring [2]. This paper presents the recent development of a real-time ammonia monitor for agricultural applications [3].
Sensor Element
The modular Air-Sense ammonia monitoring system builds on prior IOS development of an optical fiber sensor cladding, an optical grade polymer formulation with embedded indicator dye. The polymer claddings are customized for specific properties, including low scattering for low loss, miscibility with indicator dyes, and good diffusion of the target gases. Conventional film preparation methods such as spin coating, illustrated in Fig. 1, controllably coat material onto glass slides, a quick way to optimize sensor formulations before mounting sensors on the optoelectronic units. 


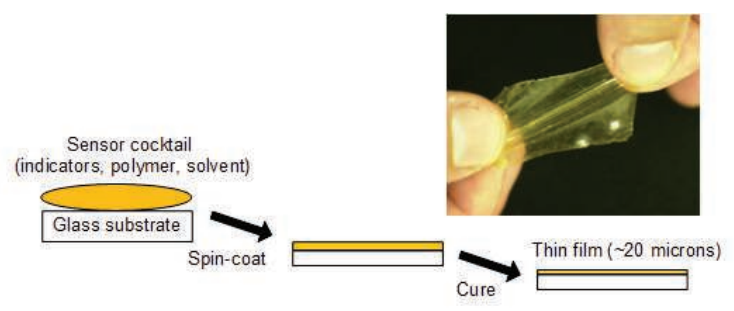

Fig. 1. Example of IOS thin film coating fabrication process on glass slide for evaluation and optimization.

Upon exposure, ammonia diffuses into the cladding and reacts with the indicator, causing the cladding to change color. In thin film testing, the color change intensity is linearly related to the gas concentration, in accordance with the Beer's Law quantification of gas concentration, as shown in Fig. 2. The fact that the optical attenuation change is due to a wavelengthspecific change in the cladding optical absorbance means that wavelengths far from the absorbance of the indicator dye are unaffected by the presence of the analyte. This mechanism makes the system self-calibrating and self-referenced.

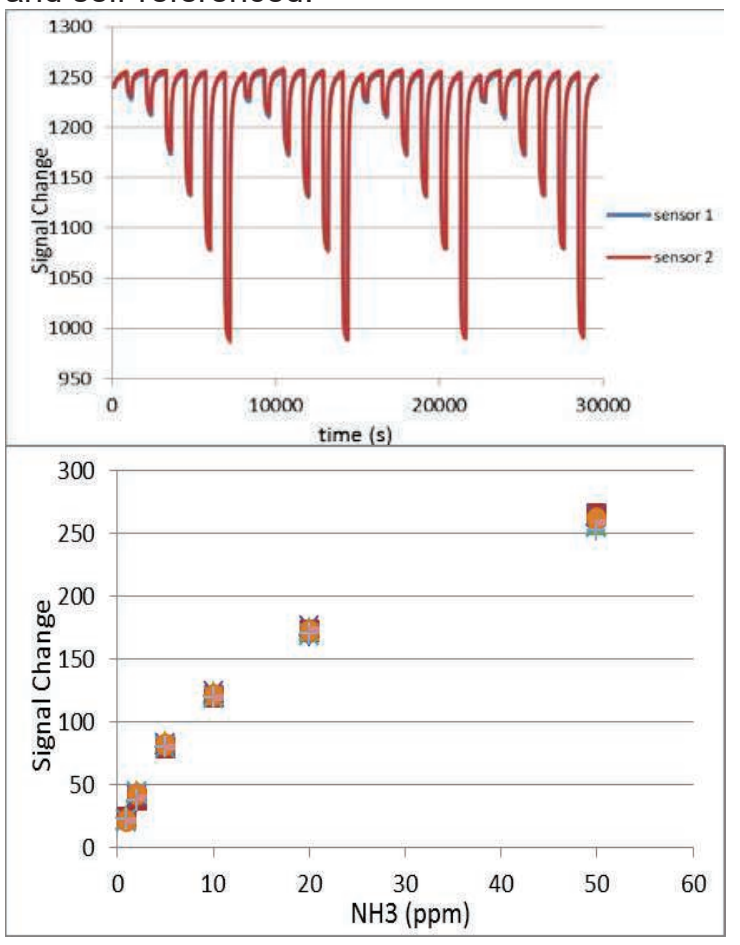

Fig. 2. (top) Signal change for two $\mathrm{NH}_{3}$ sensors upon exposure to a 1, 2, 5, 10, 20, and $50 \mathrm{ppm}$ progression (5 min. each step) with $15 \mathrm{~min}$. recovery. (bottom) Standard curve computed from the signals recorded from two sensors as a function of $\mathrm{NH}_{3}$ concentration.

Field studies have consistently played an important role in Air-Sense development. Based on the data from field deployments, a calibration profile was tuned for maximum accuracy. The duration of deployment was established based on the target application. For example, flock cycle coverage was envisioned for each sensor module, so real-time data can be logged and synchronized with ventilation systems rather than depending on point testing with a pull tube. However, our results show the units being stable through the completion of a flock cycle and poultry litter treatment (PLT). It was pointed out to us by CTB staff that PLT has a drastic poisoning effect on a number of sensors, including pull tubes [4]. Air-Sense modules were in place through four consecutive PLTs, before each flock cycle, with successful ammonia level monitoring performance.

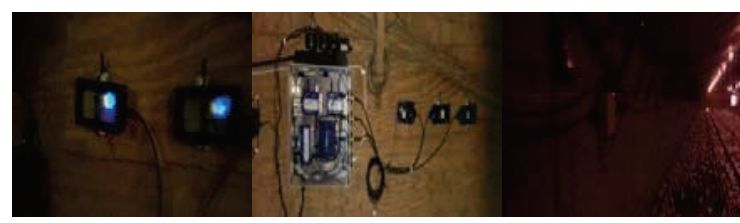

Fig. 3. Three Air-Sense units installed at CTB poultry barn in Georgia, USA.
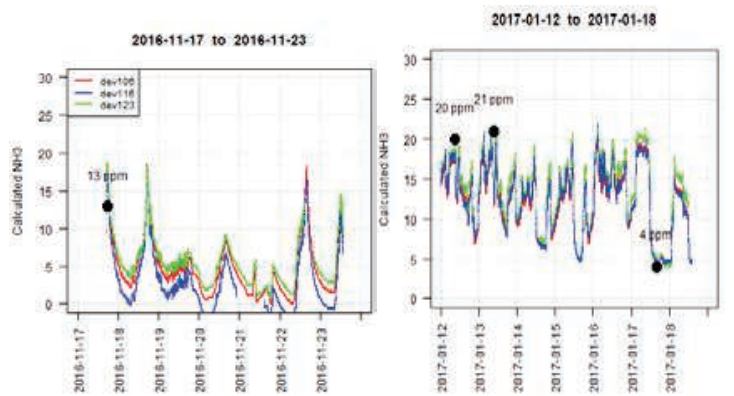

Fig. 4. Examples of weekly real-time data reported from Air-Sense modules.

\section{References}

[1] C.M. Wathes, T.G.M. Demmers, H. Xin, Ammonia Concentrations and Emissions in Livestock Facilities: Guidelines and Limits in the USA and UK, Agricultural and Biosystems Engineering Conference Proceedings and Presentations, Paper No. 034112 (2003); http://lib.dr.iastate.edu/abe_eng_conf/123.

[2] J. Arogo, P.W. Westerman, A.J. Heber, W.P. Robarge, J.J. Classen, Ammonia Emissions from Animal Feeding Operations (2002); doi: 10.1.1.484.8060.

[3] K. Janni, L.D. Jacobson, B.P. Hetchler, L.J. Johnston, Comparing Semi-Continuous Air Sampling to 24-h Bag Samples, American Society of Agricultural and Biological Engineers Annual International Meeting 1, 829-846, Paper No. 1111163 (2011); ISBN 9781618391568.

[4] D.D. Lee, D.S. Lee, Environmental Gas Sensors, IEEE Sensors Journal 1(3), 214-224 (2001); doi: 10.1109/JSEN.2001.954834. 